\title{
Stochastic Solvers for the Euler Equations
}

\author{
G. Lin, C.-H. Su and G.E. Karniadakis * \\ Division of Applied Mathematics \\ Brown University \\ Providence, RI 02912
}

In this paper we extend our previous work, first presented in, ${ }^{1}$ to handle effectively non-Gaussian processes and long-time integration in unsteady simulations of compressible flows. Specifically, we apply the generalized polynomial chaos (GPC) method to solve the one-dimensional stochastic Euler equations. We present systematic verification studies against an analytical solution of the stochastic piston problem for different correlation lengths of the time-dependent random piston motion, which may follow a Gaussian or a uniform distribution. A new multi-element decomposition of the random space is presented that provides more robustness and resolution capability, and comparisons are made against Monte Carlo simulations.

\footnotetext{
*Corresponding author, gk@dam.brown.edu

Copyright (c) 2005 by the American Institute of Aeronautics and Astronautics, Inc. All rights reserved.
} 


\section{Introduction}

$\mathrm{T}$ here has been recently an intense interest in verification and validation of large-scale simulations and in modeling uncertainty. In simulations, just like in the experiments, we often question the accuracy of the results and we construct a posteriori error bounds, but the new objective is to model uncertainty from the beginning of the simulation and not simply as an afterthought. While numerical accuracy and error control have been employed in simulations for some time now at least for the more modern discretizations, there is still an uncertainty component associated with the compressible and MHD applications, especially for high Mach number flows. Uncertainty may be present in the transport coefficients, and in the boundary conditions.

In order to deal with the uncertainties numerically, statistical and non-statistical computational strategies have been applied. The statistical approaches, e.g. Monte Carlo simulations, can be prohibitively expensive, especially for large values of standard deviation and time-dependent flows. A non-statistical approach, called polynomial chaos, is based on the homogeneous chaos theory of Wiener. ${ }^{2}$ It models uncertainty by a spectral expansion based on Hermite orthogonal polynomials in terms of Gaussian random variables. This method was applied by Ghanem and co-workers to various problems in mechanics. ${ }^{3,4}$ A broader framework, called the "generalized polynomial chaos", was introduced in. ${ }^{5,6}$ This method employs a broad family orthogonal polynomials as the expansion basis to represent non-Gaussian processes more efficiently; it includes the classical Hermite polynomial chaos as a subset.

The spectral representation of the uncertainty is based on a trial basis $\psi(\theta)$ where $\theta$ denotes the random event. For example, the temperature has the following finite-dimensional representation

$$
T(\mathbf{x}, t, \theta)=\sum_{i=0}^{N_{p}} \hat{T}_{i}(\mathbf{x}, t) \psi_{i}\left(\xi_{i}(\theta)\right),
$$

where $\hat{T}_{i}(\mathbf{x}, t)$ is the deterministic mode. The random trial basis is expressed in terms of multi-dimensional Hermite polynomials (or the generalized chaos polynomials) in terms of the random variable $\xi_{i}(\theta)$, which follows a specific probability distribution. For example, $\xi_{i}(\theta)$ may be a Gaussian function. For $\xi_{i}(\theta)$ following a uniform distribution the corresponding basis is Legendre polynomials. The polynomial trial basis constructed in this way has been termed Polynomial Chaos by Wiener. It is a functional, as it is a function of $\xi_{i}$ which is a function of the random variable $\theta \in[0,1]$.

The representation of stochastic inputs is expressed by a Karhunen-Loeve decomposition. Let $h(\mathbf{x} ; \theta)$ denote the random process and $R_{h h}(\mathbf{x}, \mathbf{y})$ the correlation function, where $x$ and $y$ are the spatial or temporal coordinates. The Karhunen-Loeve(KL) decomposition ${ }^{7}$ can be expressed as:

$$
h(\mathbf{x} ; \theta)=\bar{h}(\mathbf{x})+\sum_{i=0}^{\infty} \sqrt{\lambda_{i}} \phi_{i}(\mathbf{x}) \xi_{i}(\theta)
$$

where $\bar{h}(\mathbf{x})$ denotes the mean of the random process, and $\left\{\xi_{i}(\theta)\right\}$ is a set of uncorrelated random variables with zero mean and unit variance. Also, $\phi_{i}(\mathbf{x})$ and $\lambda_{i}$ are the eigenfunctions and eigenvalues of the correlation function, respectively, i.e.,

$$
\int_{\mathbf{D}} R_{h h}(\mathbf{x}, \mathbf{y}) \phi_{i}(\mathbf{y}) d \mathbf{y}=\lambda_{i} \phi_{i}(\mathbf{x})
$$

To test the new solvers, we have developed exact analytical solutions for the linearized Euler equations for the stochastic piston problem. ${ }^{1}$ It is a re-formulation, within the stochastic framework, of a classical aerodynamics benchmark problem that studies how small random piston motions affect shock paths. The analytical stochastic solution makes this problem an excellent benchmark for developing CFD and MHD stochastic algorithms and corresponding codes. We have applied Hermite-chaos for Gaussian random inputs and Legendre-chaos for uniform random inputs with fifth-order WENO scheme ${ }^{8}$ in space and third-order Runge-Kutta method in time. To deal with long-term integration in the stochastic Euler system, a MultiElement Generalized Polynomial Chaos (ME-GPC) method $^{9}$ was presented. The main idea of ME-GPC is to decompose the space of random inputs and apply Generalized Polynomial Chaos (GPC) in each random element. The error due to long-term integration can be effectively controlled by the adaptive random decomposition. ${ }^{10}$ 
The paper is organized as follows: In section 2, we summarize the analytical solutions from the stochastic perturbation analysis. In section 3, we present the GPC and ME-GPC stochastic solvers for Euler equations. We represent some numerical results in section 4 . We conclude in section 5 with a few remarks.

\section{Theory}

We consider a piston having a constant velocity, $U_{p}$, moving into a straight tube filled with a homogeneous gas at rest. A shock wave will be generated ahead of the piston. Fig. 1 is a sketch of piston-driven shock tube with stochastic random piston motion. We consider the perturbation component of the piston velocity $v_{p}$ as a random process with the zero mean and exponential covariance,

$$
\left.\begin{array}{c}
v_{p}(t)=\epsilon U_{p} V(t, \xi) \\
\langle V(t, \xi)\rangle=0 \\
\left\langle V\left(t_{1}, \xi\right), V\left(t_{2}, \xi\right)\right\rangle=e^{-\frac{\left|t_{1}-t_{2}\right|}{A}}
\end{array}\right\}
$$

where $A$ is the correlation time; $\epsilon$ the excitation amplitudes; and $U_{p}$ the steady piston velocity. We also define $C$ as the steady sound speed behind the shock and $S$ as the steady shock speed.

The above covariance kernel describes a Markov random process in time. The larger the value of the correlation time $A$ is the closer the random motion we pick every time step to be fully-correlated - we refer to the latter as a random variable case. On the other hand, the smaller the value of the correlation time $A$ is the closer the motion resembles white noise.

Fig. 2 is a sketch of shock paths induced by stochastic random piston motions. The distorted lines show instantaneous realization of the piston path and shock path. They are distorted due to induced reflections as sketched in the plot via the characteristic lines. Using stochastic perturbation analysis (see ${ }^{1}$ ), we obtained the exact solution for variance, $\left\langle z^{2}(\tau)\right\rangle$, of the perturbed shock paths (i.e., the square of the distance from the mean shock location).

$$
\left\langle z^{2}(\tau)\right\rangle=\left(U_{p} q S^{\prime} A / \alpha\right)^{2}\left[2 \sum_{n=1}^{\infty} \sum_{m=0}^{n-1}(-r)^{n+m} I_{n, m}(\tau)+\sum_{n=0}^{\infty} r^{2 n} I_{n, n}(\tau)\right]
$$

where $\tau=\alpha t / A$ and

$$
I_{n, m}(\tau)=\frac{2 \tau}{\beta^{m}}+\frac{1}{\beta^{n+m}}\left[e^{-\beta^{m} \tau}+e^{-\beta^{n} \tau}-1-e^{-\left(\beta^{m}-\beta^{n}\right) \tau}\right]
$$

with $m<n$ and

$$
\begin{aligned}
S^{\prime} & =\frac{d S}{d U_{p}}, k=C \frac{S+S^{\prime} U_{p}}{1+\gamma S U_{p}} \\
r & =\frac{1+k}{1-k}, q=\frac{2}{1+k} \\
\alpha & =\frac{C+U_{p}-S}{C}, \beta=\frac{C+U_{p}-S}{C+S-U_{p}}, \gamma=\frac{1}{C+S-U_{p}}
\end{aligned}
$$

For $\tau \ll 1$ (early times), the above formula can be simplified as:

$$
\left\langle z^{2}(\tau)\right\rangle \approx\left(U_{p} q S^{\prime} A / \alpha\right)^{2} \frac{\tau^{2}}{(1+r)^{2}} \text { for } \tau \ll 1 .
$$

For $\tau \gg 1$ (long time), we can neglect the exponentials in Eq. 6 to obtain:

$$
\left\langle z^{2}(\tau)\right\rangle \approx\left(U_{p} q S^{\prime} A / \alpha\right)^{2}\left[\frac{2 \tau(1-r)}{\left(1-r^{2} / \beta\right)(1+r)}-\frac{2}{\left(1-r^{2} / \beta^{2}\right)(1+r / \beta)}\right], \text { for } \tau \gg 1 .
$$

We plot in Fig. 3 the quantity $\left\langle z^{2}(\tau)\right\rangle /\left(U_{p} q S^{\prime} A / \alpha\right)^{2}$ as a function of $\tau$ given by Eq. 5 with $U_{p}=1.25$, corresponding to Mach number of the shock $M=2$. The asymptotic formula for small and large $\tau$ given in Eqs. 7 and 8 are also included in the plot. Therefore, from the two equations above, we see a qualitative change in the response versus time. At early times, the location of the path scales linearly with time; whereas at later times, the location of the path scales with square root of time (Note that $\left.\left\langle z^{2}(\tau)\right\rangle \sim(\text { Length })^{2}\right)$. 


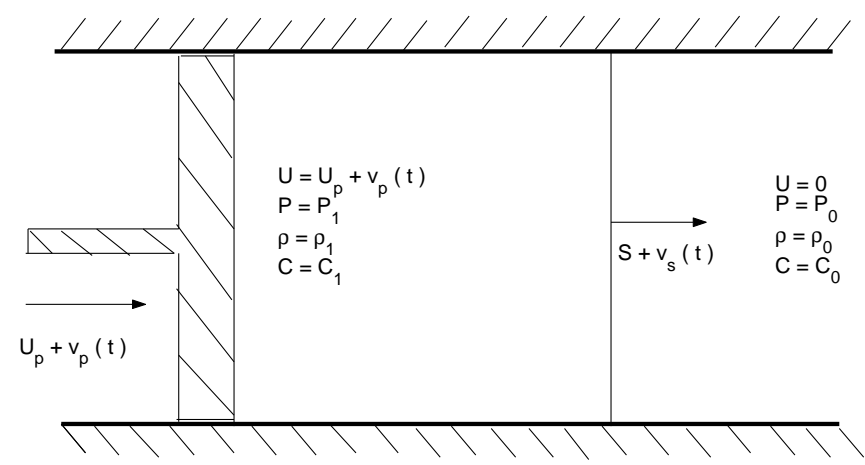

Figure 1. A sketch of piston-driven shock tube with stochastic random piston motion.

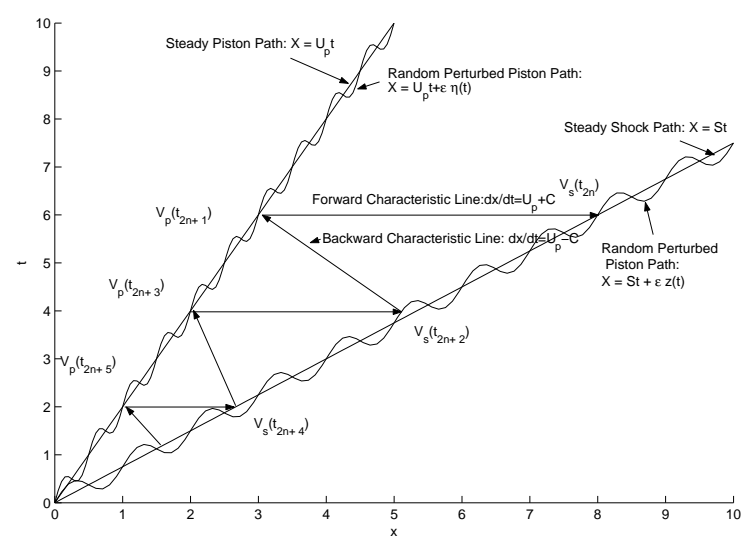

Figure 2. A sketch of shock paths induced by stochastic random piston motions.

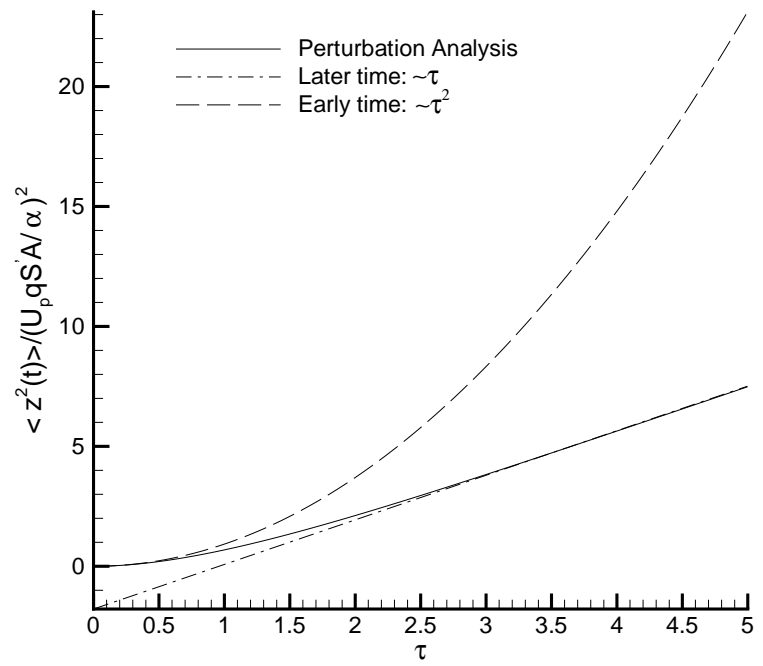

Figure 3. Normalized variance of perturbed shock paths. Solid line: perturbation analysis results, see Eq. 5; Dashed line: early-time asymptotic results from Eq. 7; Dash-Dotted line: late-time asymptotic results from Eq. 8. 


\section{Solvers}

In this section, we solve the full nonlinear Euler equations with unsteady stochastic boundary for the stochastic piston problem. A boundary-fitted coordinate approach is employed to transform the equations into a stationary framework. We perform two types of stochastic simulations to verify the analytical solutions by following a Monte Carlo approach and a polynomial chaos approach. In Monte Carlo simulations we use a Markov chain in time and also a Karhunen-Loeve decomposition to represent stochastic inputs. In polynomial chaos simulations, the representation of stochastic inputs is expressed by a Karhunen-Loeve decomposition; see references $\left({ }^{3,5}\right)$.

\section{A. Transformed Euler Equations}

We employ the one-dimensional Euler equations

$$
\begin{aligned}
\frac{\partial \rho}{\partial t}+\frac{\partial \rho u}{\partial x} & =0 \\
\frac{\partial \rho u}{\partial t}+\frac{\partial}{\partial x}\left(\rho u^{2}+p\right) & =0 \\
\frac{\partial E}{\partial t}+\frac{\partial}{\partial x}[u(p+E)] & =0
\end{aligned}
$$

where $\rho$ denotes density, $u$ is $x$ component velocity, $E$ is total energy, $m=\rho u$ is the $x$ component momentum, and $\gamma$ is the ratio of the specific heats. $p$ is pressure, where $p=(\gamma-1)\left(E-\frac{1}{2} \rho u^{2}\right)$. All flow quantities, i.e., velocity, pressure, $x$ component momentum and total energy are considered as stochastic processes. A random dimension, denoted by the parameter $\xi$, is introduced in addition to the spatial-temporal dimensions $(x, t)$, thus

$$
u=u(x, t ; \xi), \quad p=p(x, t ; \xi), \quad m=m(x, t ; \xi), \quad E=E(x, t ; \xi)
$$

To deal with the moving boundaries of the piston, we consider a boundary-fitted coordinate approach for the piston problem. By attaching the coordinate system to the piston, the piston appears stationary in time (with respect to that coordinate system). We define two coordinate systems $(x, t)$ and $(\zeta, \tau)$, where $(x, t)$ is the original coordinate system and $(\zeta, \tau)$ is the transformed one. The mapping between the two systems is:

$$
\left\{\begin{array}{l}
\zeta=x-\int_{0}^{\tau} u_{p}\left(\tau_{1}, \xi\right) d \tau_{1}, \\
\tau=t
\end{array}\right.
$$

In one-dimensional flow, this simply reduces to the new velocity $v$ being shifted by the reference frame velocity, i.e.,

$$
v=u-u_{p}(t, \xi) .
$$

It is worth noting that this mapping is stochastic when the piston motion is random and needs to be represented by the chaos expansion as well. The compressible Euler equations (9) with stochastic timedependent boundary conditions are transformed into:

$$
\begin{aligned}
\frac{\partial \rho}{\partial \tau}+\frac{\partial \rho v}{\partial \zeta} & =0 \\
\frac{\partial \rho v}{\partial \tau}+\frac{\partial}{\partial \zeta}\left(\rho v^{2}+p\right) & =-\rho \frac{\partial u_{p}}{\partial \tau} \\
\frac{\partial E^{\prime}}{\partial \tau}+\frac{\partial}{\partial \zeta}\left[v\left(p+E^{\prime}\right)\right] & =-\rho v \frac{\partial u_{p}}{\partial \tau}
\end{aligned}
$$

We model the piston velocity as a process

$$
u_{p}=U_{p}+v_{p}=U_{p}(1+\epsilon V(t, \xi)),
$$

where $v_{p}$ is perturbation component of the piston velocity. Specifically, we consider different representations of the stochastic inputs $v_{p}$ corresponding to a random process with zero mean and exponential covariance; 
see Eq. 4. It expresses the rate at which the correlation decays between two time instants of the process. A corresponding Markov chain is employed to represent discretely the exponential kernel as follows,

$$
\left\{\begin{array}{l}
V_{0}=\xi_{0}, \\
V_{1}=b V_{0}+f \xi_{1}, \\
\cdots \\
V_{i+1}=b V_{i}+f \xi_{i+1},
\end{array}\right.
$$

where $b=e^{\frac{-\Delta t}{A}}, f=\sqrt{1-b^{2}}$. In the Monte Carlo simulation, a random piston velocity $u_{p}=U_{p}\left(1+\epsilon V_{i}(t, \xi)\right)$ is selected from the above Markov chain as a stochastic input at each time step $t_{i}$. In the polynomial chaos representation we employ the Wiener-Hermite expansions for all conservative and derived stochastic variables. The representation of stochastic inputs is expressed by a Karhunen-Loeve decomposition.

\section{B. Generalized Polynomial Chaos (GPC)}

Polynomial chaos is based on the homogeneous chaos theory of Wiener. ${ }^{2}$ It models a stochastic process by a spectral expansion based on Hermite orthogonal polynomials in terms of Gaussian random variables. GPC was proposed in ${ }^{6}$ and employs more types of orthogonal polynomials from the Askey scheme. It is a generalization of the original Wiener's Hermite-chaos and can deal with non-Gaussian random inputs more efficiently. A general second-order random process $T(\xi)$ can be expressed by GPC as,

$$
T(x, t, \theta)=\sum_{i=0}^{N_{p}} \hat{T}_{i}(x, t) \phi_{i}\left(\xi_{i}(\theta)\right),
$$

where the family $\left\{\phi_{i}\right\}$ is an orthogonal basis with orthogonality relation,

$$
\left\langle\phi_{i}, \phi_{j}\right\rangle=\left\langle\phi_{i}^{2}\right\rangle \delta_{i j}
$$

where $\delta_{i j}$ is the Kronecker delta, and $\langle\cdot, \cdot\rangle$ denotes the ensemble average. Here the ensemble average can be defined as the inner product in the Hibert space in terms of the random vector $\boldsymbol{\xi}=\left(\xi_{1}, \xi_{2}, \ldots, \xi_{d}\right)$,

$$
\langle f(\boldsymbol{\xi}), g(\boldsymbol{\xi})\rangle=\int f(\boldsymbol{\xi}) g(\boldsymbol{\xi}) w(\boldsymbol{\xi}) d \boldsymbol{\xi}
$$

or

$$
\langle f(\boldsymbol{\xi}), g(\boldsymbol{\xi})\rangle=\sum_{\boldsymbol{\xi}} f(\boldsymbol{\xi}) g(\boldsymbol{\xi}) w(\boldsymbol{\xi})
$$

in the discrete case, where $w(\boldsymbol{\xi})$ denotes the weight function. For a certain random vector $\boldsymbol{\xi}$, the GPC basis $\left\{\phi_{i}\right\}$ can be chosen in such a way that its weight function has the same form as the probability distribution function of $\boldsymbol{\xi}$. The total number of basis modes $N_{p}$ is determined by the dimensionality of the chaos expansion $d$ and the highest order p of the polynomials $\phi_{i}$, where

$$
N_{p}=\frac{(d+p) !}{(d ! p !)}-1
$$

The most important aspect of the above chaos expansion is that the random processes have been decomposed into a set of deterministic functions in the spatial-temporal variables multiplied by the random basis polynomials which are independent of these variables. Substituting above into one-dimensional Euler equations we obtain the following equations,

$$
\begin{array}{r}
\sum_{i=0}^{N_{p}} \frac{\partial}{\partial \tau}\left(\begin{array}{c}
\hat{\rho}_{i} \phi_{i} \\
\hat{m}_{i} \phi_{i} \\
\hat{E}_{i} \phi_{i}
\end{array}\right)+\sum_{i=0}^{N_{p}} \frac{\partial}{\partial \zeta}\left(\begin{array}{c}
\hat{m}_{i} \phi_{i} \\
\sum_{j=0}^{N_{p}} \hat{m}_{i} \hat{v}_{j} \phi_{i} \phi_{j}+\hat{p}_{i} \phi_{i} \\
\sum_{j=0}^{N_{p}}\left(\hat{E}_{i}+\hat{p}_{i}\right) \hat{v}_{j} \phi_{i} \phi_{j}
\end{array}\right) \\
=\sum_{i=0}^{N_{p}}\left(\begin{array}{c}
0 \\
-\sum_{j=0}^{N_{p}} \hat{\rho}_{i} \frac{\partial \hat{u}_{p j}}{\partial \tau} \phi_{i} \phi_{j} \\
-\sum_{j=0}^{N_{p}} \hat{m}_{i} \frac{\partial \hat{u}_{p j}}{\partial \tau} \phi_{i} \phi_{j}
\end{array}\right)
\end{array}
$$

6 of 13 
A Galerkin projection of the above equations onto each polynomial basis $\phi_{i}$ is then conducted in order to ensure the error is orthogonal to the functional space spanned by the finite-dimensional basis $\phi_{0}, \ldots, \phi_{N_{p}}$. By projecting with $\phi_{k}$ for each $k=0, \ldots, N_{p}$ and employing the orthogonality relation, we obtain for each $k=0, \ldots, N_{p}$,

$$
\begin{aligned}
& \frac{\partial}{\partial \tau}\left(\begin{array}{c}
\hat{\rho}_{k} \\
\hat{m}_{k} \\
\hat{E}_{k}
\end{array}\right)+\frac{\partial}{\partial \zeta}\left(\begin{array}{c}
\hat{m}_{k} \\
\frac{1}{<\phi_{k}^{2}>} \sum_{i=0}^{N_{p}} \sum_{j=0}^{N_{p}} \hat{m}_{i} \hat{v}_{j} e_{i, j, k}+\hat{p}_{k} \\
\frac{1}{<\phi_{k}^{2}>} \sum_{i=0}^{N_{p}} \sum_{j=0}^{N_{p}}\left(\hat{E}_{i}+\hat{P}_{i}\right) \hat{v}_{j} e_{i, j, k}
\end{array}\right)= \\
&\left(\begin{array}{c}
-\epsilon \\
-\frac{\epsilon U_{p}}{<\phi_{k}^{2}>} \sum_{i=0}^{N_{p}} \sum_{j=1}^{d} \hat{\rho}_{i} \sqrt{\lambda_{j}} \frac{\partial f_{j}}{\partial \tau} e_{i, j, k} \\
-\frac{\epsilon U_{p}}{<\phi_{k}^{2}>} \sum_{i=0}^{N_{p}} \sum_{j=1}^{d} \hat{m}_{i} \sqrt{\lambda_{j}} \frac{\partial f_{j}}{\partial \tau} e_{i, j, k}
\end{array}\right)
\end{aligned}
$$

where $f_{j}(\tau)$ and $\lambda_{j}$ are the eigenfunctions and eigenvalues of the correlation function and $e_{i, j, k}=<\phi_{i} \phi_{j} \phi_{k}>$. Both $\left\langle\phi_{k}^{2}\right\rangle$ and $e_{i, j, k}$ can be evaluated analytically during the pre-processing stage. The set of equations consists of $\left(N_{p}+1\right)$ system of deterministic 'Euler-like' equations for each random mode coupled through the convective terms.

Discretization in space and time can be carried out by any conventional method. Here we employ the fifth-order WENO method in spatial discretization in order to capture the shock location accurately. A third-order TVD Runge-Kutta method is employed in time; see details about WENO method in. ${ }^{8}$

After solving the deterministic expansion coefficients, we obtain the analytical form (in random space) of the solution process. Since the shock location is not a physical variable of the Euler equations, Monte Carlo simulation is employed on the stochastic solutions in order to carry out the shock path analysis. A shock-location-capture routine is employed to find the shock location at each time step. We solve the above 'Euler-like' equations based on the coordinate system attached to the piston. We then have to transform the solutions back to the original coordinate system:

$$
\left\{\begin{array}{l}
x=\zeta+\int_{0}^{\tau} u_{p}\left(\tau_{1}, \xi\right) d \tau_{1}, \\
t=\tau
\end{array}\right.
$$

where $x$ and $\zeta$ are the shock location in the original coordinate system and the shock location in the transformed coordinate system, respectively. The shock location $x$ includes the static part and the perturbed part. The perturbed shock location $z(\tau)=x-S t$. The mean and variance of the perturbed shock paths are obtained as,

$$
\left\{\begin{array}{l}
\bar{z}(\tau)=\frac{1}{n} \sum_{i=0}^{n} z_{i}(\tau) \\
<z^{2}(\tau)>=\frac{1}{n-1} \sum_{i=0}^{n}\left(z_{i}(\tau)-\bar{z}(\tau)\right)^{2}
\end{array}\right.
$$

where $\mathrm{n}$ is the total number of sample space in Monte Carlo simulations.

\section{Multi-Element Generalized Polynomial Chaos (ME-GPC)}

GPC can achieve exponential convergence, however the error of GPC will increase gradually with time. Therefore GPC may lose its effectiveness and the convergence may fail for problems requiring long-term integration. In this section, a multi-element generalized polynomial chaos scheme is inplemented to solve the stochastic piston problem. Next, we summarize the theory developed in ${ }^{9} .^{10}$

Let us denote $\boldsymbol{\xi}=\left(\xi_{1}, \xi_{2}, \cdots, \xi_{d}\right)$ to be a $d$-dimensional random vector and $\mathcal{P}\left(\Omega_{i}, \mathcal{A}_{i}, P_{i}\right)$ be the probability space of $\xi_{i}$. We assume that $\xi_{i}$ are uniform random variables defined as $\xi_{i}: \Omega_{i} \mapsto[-1,1]$ with a constant PDF $f_{i}=\frac{1}{2}$. Thus, $\boldsymbol{\xi}$ is a random vector defined as $\boldsymbol{\xi} \mapsto B$ with a constant $\operatorname{PDF} f=\left(\frac{1}{2}\right)^{d}$, where $B=[-1,1]^{d}$. 
$B$ can be decomposed into a set $\mathbf{D}$ with $N$ non-overlapping elements

$$
\mathbf{D}=\left\{\begin{array}{l}
B_{k}=\left[a_{k, 1}, b_{k, 1}\right] \times\left[a_{k, 2}, b_{k, 2}\right] \times \cdots \times\left[a_{k, d}, b_{k, d}\right], \\
B=\bigcup_{k}^{N} B_{k}, \\
B_{k_{1}} \cap B_{k_{2}}=\emptyset, \text { if } k_{1} \neq k_{2},
\end{array}\right.
$$

where $k, k_{1}, k_{2}=1,2, \cdots, N$. In each element, a new $d$-dimensional uniform random vector is defined to be

$$
\boldsymbol{\xi}_{k}=g_{k}(\boldsymbol{\xi})=\left(\xi_{k, 1}, \xi_{k, 2}, \cdots, \xi_{k, d}\right): \Omega_{k} \mapsto[-1,1]^{d}
$$

with a constant PDF $f^{k}=\left(\frac{1}{2}\right)^{d}$. The new d-dimensional random vector can be mapped from a uniform random vector as following,

$$
g_{k}(\boldsymbol{\xi}): \xi_{i}=\frac{b_{k, i}-a_{k, i}}{2} \xi_{k, i}+\frac{b_{k, i}+a_{k, i}}{2}, \quad i=1,2, \cdots, d .
$$

The determinant of the Jacobian is the ratio of the volume of element $k$ over the volume of the whole random space

$$
\operatorname{det}\left|\frac{\partial \boldsymbol{\xi}}{\partial \boldsymbol{\xi}_{k}}\right|=\prod_{i=1}^{d} \frac{b_{k, i}-a_{k, i}}{2},
$$

In each element $k$, we implement GPC and solve the system according to $\boldsymbol{\xi}_{k}$. The Karhunen-Loeve (K-L) decomposition for each element $k$ can be expressed as:

$$
u\left(\mathbf{t} ; \xi_{k}\right)=\bar{u}(\mathbf{t})+\sum_{i=1}^{d} \sqrt{\lambda_{i}} f_{i}(\mathbf{t})\left(\frac{b_{k, i}-a_{k, i}}{2} \xi_{k, i}+\frac{b_{k, i}+a_{k, i}}{2}\right)
$$

After we obtain the approximation $\hat{u}_{k}\left(\boldsymbol{\xi}_{k}\right)$ for each element $k(k=1,2, \cdots, N)$, we can reconstruct the $m$-th moment of $u(\boldsymbol{\xi})$ on the entire random domain,

$$
\mu_{m}(u(\boldsymbol{\xi}))=\int_{B} u^{m}(\boldsymbol{\xi})\left(\frac{1}{2}\right)^{d} d \boldsymbol{\xi}=\sum_{k=1}^{N} \int_{[-1,1]^{d}} \hat{u}_{k}^{m}\left(\boldsymbol{\xi}_{k}\right)\left(\frac{1}{2}\right)^{d} \operatorname{det}\left|\frac{\partial \boldsymbol{\xi}}{\partial \boldsymbol{\xi}_{k}}\right| d \boldsymbol{\xi}_{k} .
$$

Let us assume that a general second-order random process $u$ can be expressed by GPC expansion at random space in each element $k$,

$$
u_{k}\left(\boldsymbol{\xi}_{k}\right)=\sum_{j=0}^{N_{p}} \hat{u}_{k, j} \phi\left(\xi_{j}\right),
$$

where $p$ is the highest order of polynomial chaos and the total number of basis modes $N_{p}$ is given in Eq. 19 .

The local mean $\bar{u}_{k, p}$ and local variance $\sigma_{k, p}^{2}$ can be easily obtained from the orthogonality of GPC with order $p$

$$
\left\{\begin{array}{l}
\bar{u}_{k, p}=\hat{u}_{k, 0}, \\
\sigma_{k, p}^{2}=\sum_{j=1}^{N_{p}} \hat{u}_{k, j}^{2}\left\langle\phi_{j}^{2}\right\rangle,
\end{array}\right.
$$

The global mean $\bar{u}$ and the global variance $\sigma^{2}$ can be expressed as

$$
\left\{\begin{array}{l}
\bar{u}=\sum_{k=1}^{N} \hat{u}_{k, 0} \operatorname{det}\left|\frac{\partial \boldsymbol{\xi}}{\partial \boldsymbol{\xi}_{k}}\right|, \\
\sigma^{2}=\sum_{k=1}^{N}\left[\sigma_{k, p}^{2}+\left(\hat{u}_{k, 0}-\bar{u}\right)^{2}\right] \operatorname{det}\left|\frac{\partial \boldsymbol{\xi}}{\partial \boldsymbol{\xi}_{k}}\right|,
\end{array}\right.
$$


For each element $k$, Since

$$
\left\{\begin{array}{l}
\left(\rho \frac{\partial u_{p}}{\partial \tau}\right)_{k}=-\epsilon U_{p} \sum_{i=0}^{N_{p}} \sum_{j=1}^{d} \hat{\rho}_{i} \phi_{i} \sqrt{\lambda_{j}} \frac{\partial f_{j}}{\partial \tau}\left(\frac{b_{k, j}-a_{k, j}}{2} \xi_{k, j}+\frac{b_{k, j}+a_{k, j}}{2}\right), \\
\left(m \frac{\partial u_{p}}{\partial \tau}\right)_{k}=-\epsilon U_{p} \sum_{i=0}^{N_{p}} \sum_{j=1}^{d} \hat{m}_{i} \phi_{i} \sqrt{\lambda_{j}} \frac{\partial f_{j}}{\partial \tau}\left(\frac{b_{k, j}-a_{k, j}}{2} \xi_{k, j}+\frac{b_{k, j}+a_{k, j}}{2}\right),
\end{array}\right.
$$

Eq. 21 is modified for each element $k$ as:

$$
\begin{gathered}
\frac{\partial}{\partial \tau}\left(\begin{array}{c}
\hat{\rho}_{k} \\
\hat{m}_{k} \\
\hat{E}_{k}
\end{array}\right)+\frac{\partial}{\partial \zeta}\left(\begin{array}{c}
\hat{m}_{k} \\
\frac{1}{<\phi_{k}^{2}>} \sum_{i=0}^{N_{p}} \sum_{j=0}^{N_{p}} \hat{m}_{i} \hat{v}_{j} e_{i, j, k}+\hat{p}_{k} \\
\frac{1}{<\phi_{k}^{2}>} \sum_{i=0}^{N_{p}} \sum_{j=0}^{N_{p}}\left(\hat{E}_{i}+\hat{P}_{i}\right) \hat{v}_{j} e_{i, j, k}
\end{array}\right)= \\
0 \\
\left(\begin{array}{c}
0 \\
-\frac{\epsilon U_{p}}{<\phi_{k}^{2}>} \sum_{i=0}^{N_{p}} \sum_{j=1}^{d} \hat{\rho}_{i} \sqrt{\lambda_{j}} \frac{\partial f_{j}}{\partial \tau}\left(\frac{b_{k, j}-a_{k, j}}{2} e_{i, j, k}+\frac{b_{k, j}+a_{k, j}}{2} e_{i, 0, k}\right) \\
-\frac{\epsilon U_{p}}{<\phi_{k}^{2}>} \sum_{i=0}^{N_{p}} \sum_{j=1}^{d} \hat{m}_{i} \sqrt{\lambda_{j}} \frac{\partial f_{j}}{\partial \tau}\left(\frac{b_{k, j}-a_{k, j}}{2} e_{i, j, k}+\frac{b_{k, j}+a_{k, j}}{2} e_{i, 0, k}\right)
\end{array}\right)
\end{gathered}
$$

\section{Stochastic Simulations}

We now present some results for the following conditions: Behind the shock we impose a steady piston velocity $U_{p}=1.25$ (normalized by the sound speed ahead of the shock), i.e., corresponding to Mach number of the shock $M=2$. Ahead of the shock the sound speed is $C_{o}=1$ and the pressure is $P=1$.

First, we consider the piston under small deterministic time-dependent motions $v_{p}=\epsilon U_{p} \sin (\omega t)$. In Fig. 4, we plot the perturbed shock paths induced by small deterministic time-dependent piston motions $v_{p}=\varepsilon U_{p} \sin (\omega t)$. Good agreement is observed between the numerical results and the analytical solution.

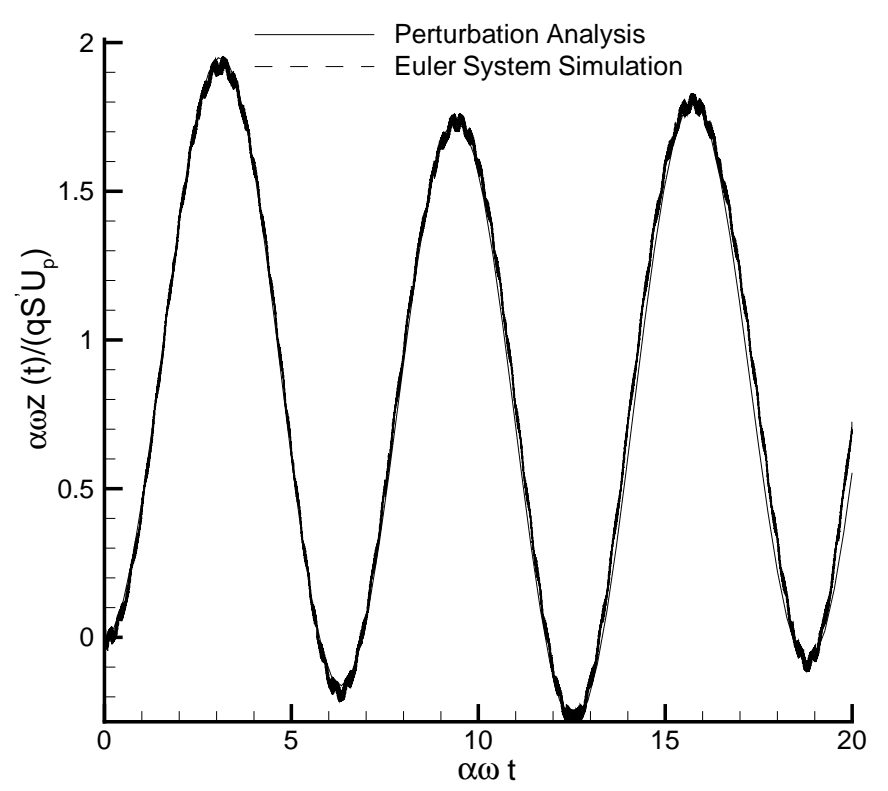

Figure 4. Shock paths induced by small deterministic time-dependent piston motions. Solid line: perturbed shock path from perturbation analysis; Dashed line: perturbed shock path obtained directly from Euler system simulation.

Fig. 5 shows results from Hermite-chaos and Legendre-chaos simulations corresponding to piston motions described by a Gaussian and uniform random variable respectively, i.e. a fully-correlated stochastic process 
whereby $A \rightarrow \infty$. The numerical results of Hermite-chaos and Legendre-chaos simulations match quite closely with the exact analytical solution (the variance of the shock location grows quadratically with time) even over a more than two-orders of magnitude change in the value of the variance. This verifies the convergence of Hermite-chaos and Legendre-chaos for this case. Fig. 6 shows the variance of the perturbed shock paths for $\epsilon=0.01$ and $A=1$ obtained from Markov-Chain Monte Carlo simulation and Karhunen-Loeve Monte Carlo simulations with stochastic dimensions $d=3,6,15,50$ and 100. The results from Markov-Chain Monte Carlo simulation exactly matches the analytical solution. Since the stochastic dimensions $d=3,6$ is not sufficient to represent the stochastic input, the numerical results obtained from Karhunen-Loeve Monte Carlo simulations with stochastic dimensions $d=3,6$ deviate from the analytical solution, especially in the early time. For the stochastic dimensions $d=15,50$ and 100, the results better approximate the analytical solution. Fig. 7 shows results obtained by Hermite-chaos method for Gaussian random inputs and Legendrechaos method for uniform random inputs, corresponding to piston motions described by a random process with amplitude $\epsilon=0.01$ and correlation time $A=1$. In the Hermite-chaos and Legendre-chaos simulations, the number of stochastic dimensions of random input is changed from $d=3,6$ to 100 ( $\mathrm{d}$ is also the number of Karhunen-Loeve modes for representing the stochastic piston motion). By increasing the dimensions of random input, the polynomial chaos simulations agree better with the analytical solution longer.

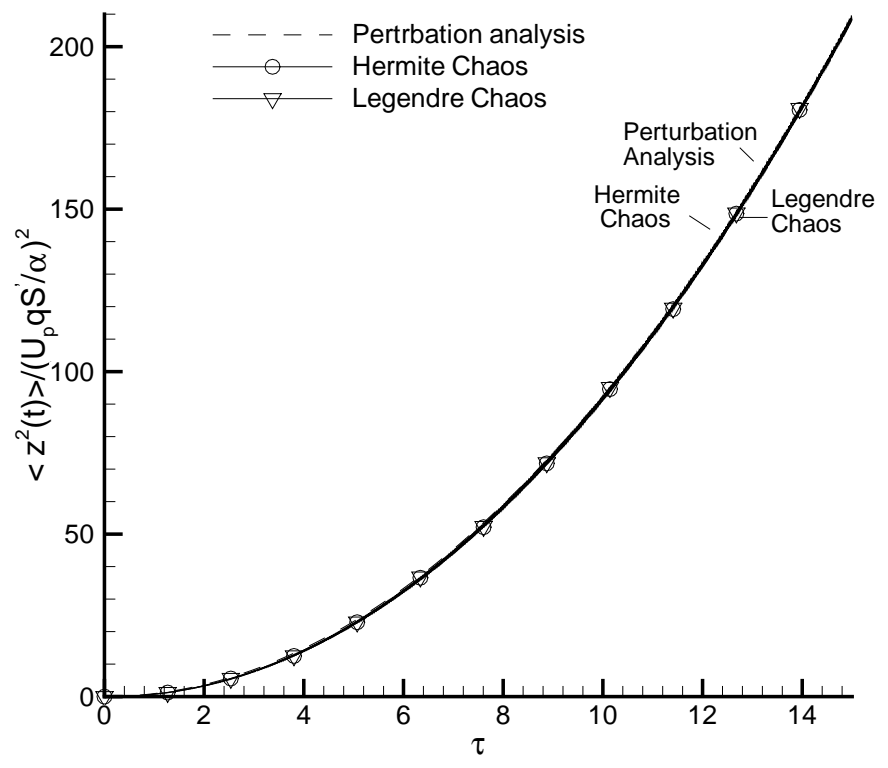

Figure 5. Variance of the perturbed shock paths for a random variable (fully-correlated kernel, $A \rightarrow \infty$ ) with amplitude $\epsilon=0.3$. Dash-Dotted line: analytical solution from perturbation analysis; Solid line: numerical results from Hermitechaos and Legendre-chaos simulations.

Fig. 8 shows results from GPC and ME-GPC (Elements $N=2,8,64$ ) simulations corresponding to piston motions described by a random process with amplitude $\epsilon=0.01$ and correlation time $A=1$. For $N=2$, only the first random dimension $\xi_{1}$, with the largest eigenvalue in K-L decomposition, is decomposed into two elements. For $N=8$ and $N=64$, the first three and all the six of the random dimensions are decomposed into two elements, respectively. The GPC and ME-GPC simulation overlap each other in the early time. However, the ME-GPC simulations perform better than the GPC simulation, especially at later time, as we increase the number of the random elements. For 64 elements, the ME-GPC numerical results show better approximation of the analytical solution in the early time.

\section{Summary and Discussion}

Developing new algorithms for uncertainty quantification requires new benchmark problems for verification of the accuracy and efficiency of the corresponding numerical solvers. Here we have presented an analytical solution of the stochastic piston problem, first derived in, ${ }^{1,11}$ which is appropriate for testing 


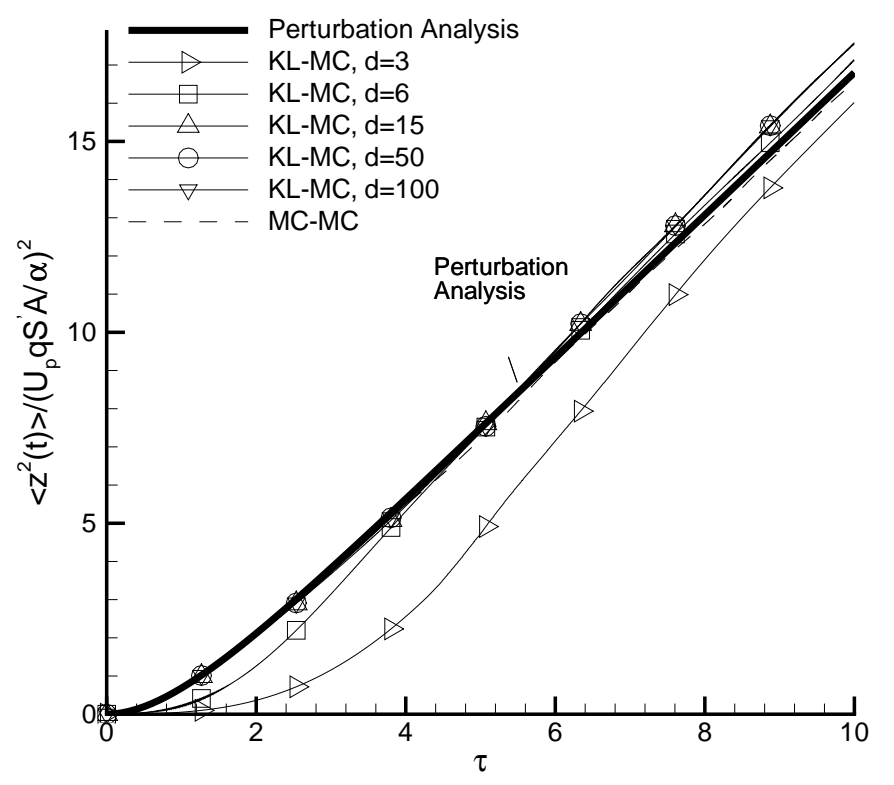

Figure 6. Variance of the perturbed shock paths for $\epsilon=0.01$ and $A=1$ obtained from Markov-Chain Monte Carlo simulation and Karhunen-Loeve Monte Carlo simulations with stochastic dimensions $d=3,6,15,50$ and 100 .

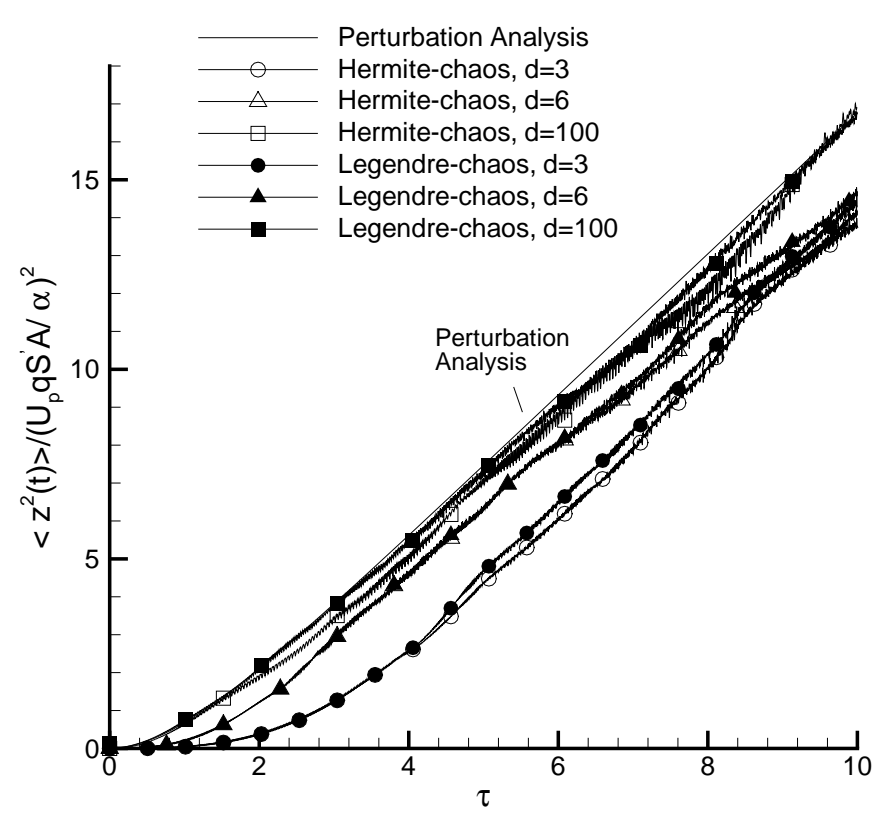

Figure 7. Variance of the perturbed shock paths for $\epsilon=0.01$ and $A=1$ obtained by Hermite-chaos method for Guassian random inputs and Legendre-chaos method for uniform random inputs with stochastic dimensions $d=3,6$ and 100 . 


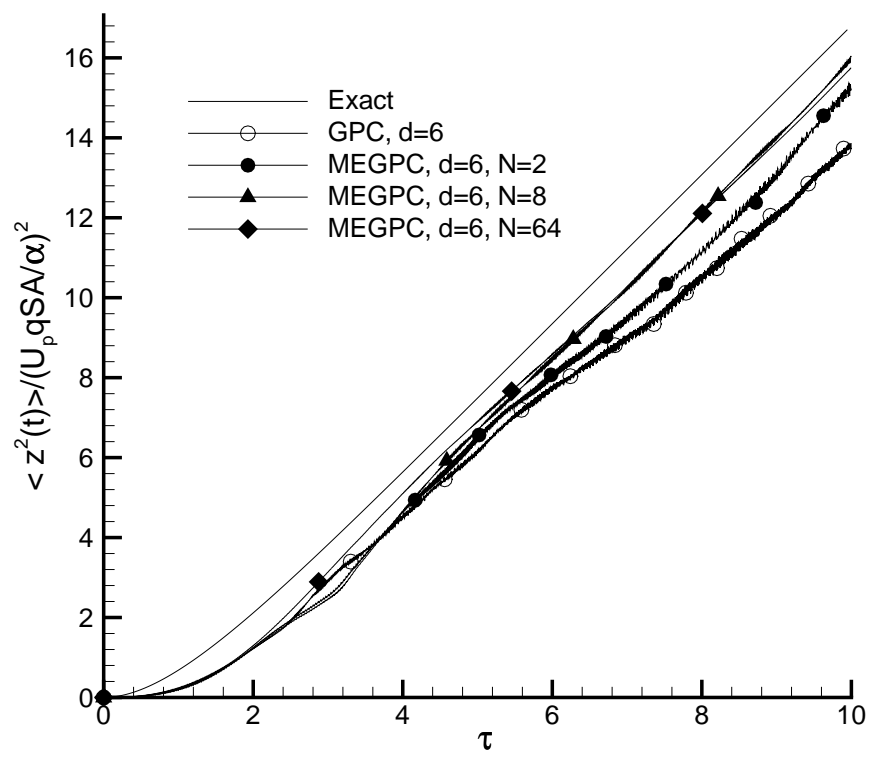

Figure 8. Variance of the perturbed shock paths for $\epsilon=0.01$ and $A=1$ obtained from GPC and ME-GPC (Elements $N=2,8,64)$ simulations with stochastic dimensions $d=6$.

time-dependent stochastic solvers for the Euler equations. The solution shows that the variance of the perturbed-shock location due to the random piston motion grows quadratically in time initially and switches to linear growth at later times.

We then presented numerical results based on Markov Chain Monte Carlo simulations as well as two different non-statistical approaches based on Wiener-Askey expansions and concepts of generalized polynomial chaos (GPC). ${ }^{5,6}$ In the first approach, global spectral-like expansions are employed to represent the stochastic solution with Hermite and Legendre polynomial functionals as trial basis that model Gaussian and uniform distributions of the random piston motion, respectively. In the second approach, the random space is decomposed into multiple elements and in each element a Legendre-chaos expansion is used, following the algorithms developed in..$^{9,10}$ These two approaches are similar to the spectral and spectral element methods for deterministic problems but the theoretical setting is in the $L_{2}$ space.

For random piston motions with amplitudes up to $20 \%$ of the steady motion, there is a good agreement of the numerical results with the analytical solutions from the stochastic perturbation analysis. This verifies the accuracy of the numerical solvers and also of the stochastic perturbation analysis. In particular, we found that for a poorly correlated stochastic piston motion represented with a Karhunen-Loeve expansion, we need high dimensionality $(d \geq 100)$ in order to achieve good agreement. The multi-element approach is effective as it reduces errors in long-time integration but also produces more accurate results than the global GPC approach at early times. A more effective approach would be to employ an adaptive scheme following, for example, the magnitude of the variance in each element and refine on-the-fly beyond a certain threshold as it was demonstrated in ${ }^{9}$ for simpler problems.

With regards to computational efficiency, GPC is more efficient than Monte Carlo simulation when the correlation length of the stochastic input is relatively large. However, for small correlation lengths where accurate representation of the stochastic input may require Karhunen-Loeve expansions with high dimensionality accelerated Monte Carlo simulations are more efficient than GPC.

\section{Acknowledgements}

This work was supported by the Computational Mathematics program of AFOSR. Computations were performed at Brown's TCASCV, NCSA and NPACI's facilities. 


\section{References}

${ }^{1}$ G. Lin, C.H. Su, and G.E. Karniadakis. The Stochastic Piston Problem. Proceedings of the National Academy of Sciences of the United States of America, 101(45):15840-15845, 2004.

${ }^{2}$ N. Wiener. The Homogeneous Chaos. Amer. J. Math., 60:897-936, 1938.

${ }^{3}$ R. G. Ghanem and P. Spanos. Stochastic Finite Eelements: A Spectral Approach. Springer-Verlag, New York, 1991.

${ }^{4}$ R. G. Ghanem and J. Red-Horse. Propagation of Uncertainty in Complex Physical Systems Using a Stochastic Finite Elements Approach. Physica D, 133:137-144, 1999.

${ }^{5}$ D. Xiu and G. E. Karniadakis. The Wiener-Askey Ppolynomial Chaos for Stochastic Differential Equations. SIAM J. Sci. Comput., 24(2):619-644, 2002.

${ }^{6}$ D. Xiu and G.E. Karniadakis. Modeling Uncertainty in Flow Simulations via Generalized Polynomial Chaos. J. Comput. Phys., 187:137-167, 2003.

${ }^{7}$ M. Loeve. Probability Theory, fourth ed. Springer-Verlag, New York, 1977. 1996.

${ }^{8}$ G.-S. Jiang and C.-W. Shu. Efficient Implementation of Weighted ENO Schemes. J. of Comput. Phys., 126:202-228,

${ }^{9}$ X.L. Wan and G.E. Karniadakis. Beyond Wiener-Askey Expansions: Handling Arbitrary PDFS. Journal of Scientific Computing, Submitted, 2004.

${ }^{10}$ X.L. Wan and G.E. Karniadakis. An Adaptive Multi-Element Generalized Polynomial Chaos Method for Stochastic Differential Equations. J. Comput. Phys., Submitted, 2004.

${ }^{11}$ C.-H. Su. Shock Paths Induced by Small Random Piston Motions: A Perturbation Analysis. Report, Center for Fluid Mechanics, Brown University, 2004. 